\title{
Determination of Specific Surface of Rock Grains by 2D Imaging
}

\author{
Arash Rabbani, ${ }^{1}$ Saeid Jamshidi, ${ }^{2}$ and Saeed Salehi ${ }^{3}$ \\ ${ }^{1}$ Petroleum University of Technology, Tehran 1453953153, Iran \\ ${ }^{2}$ Department of Chemical and Petroleum Engineering, Sharif University of Technology, Tehran 1136511155, Iran \\ ${ }^{3}$ Petroleum Engineering Department, College of Engineering, University of Louisiana at Lafayette, Lafayette, LA 70504, USA
}

Correspondence should be addressed to Saeed Salehi; saeads@gmail.com

Received 21 October 2013; Revised 26 March 2014; Accepted 26 March 2014; Published 17 April 2014

Academic Editor: Salvatore Gambino

Copyright (C) 2014 Arash Rabbani et al. This is an open access article distributed under the Creative Commons Attribution License, which permits unrestricted use, distribution, and reproduction in any medium, provided the original work is properly cited.

\begin{abstract}
Specific surface is an important parameter for predicting permeability of porous rocks. Many digital methods have been invented to extract the rock properties via imaging such as Micro-CT. With utilizing 3D volume data, this helps in precise investigation; however, it is neither economically efficient nor can be applied for different situations. In this study, a new approach is developed to estimate rock specific surface using $2 \mathrm{D}$ thin section images with micron resolution. One specific conclusion of this study is that there is specific ratio between the specific perimeter of $2 \mathrm{D}$ images and the specific surface in the $3 \mathrm{D}$ real rock structure. To further investigate this ratio several 3D blocks of rock volume data have been virtually cut in every possible angle and the value of specific perimeter calculated for each obtained 2D thin section. Finally, the predicted value of specific surface for 6 rock types is compared with the real values calculated from the original 3D data. Result indicates acceptable precision of this approach for sandstone rocks whereas not applicable for carbonate rocks.
\end{abstract}

\section{Introduction}

Specific surface area of a porous material is defined as the interstitial surface area of pores per unit of bulk volume or solid volume of the porous material. Specific surface is critical parameter considering fluid permeability of a porous material [1-3]. Since specific surface is the ratio of area to volume, it has dimension of $\mathrm{L}^{-1}$. The specific surface of porous granular media is defined as follows [3]:

$$
\text { Specific Surface }=S=\frac{\text { Surface area of grains }}{\text { Volume of rock grains }} \text {. }
$$

Several methods for estimating specific surface of porous materials have been discussed in the literature until now. Collins [1] is one of the first pioneers of using these methods for specific surface measurement. Collins categorized the methods of specific surface determination into three main groups as follows: (A) adsorption measurement of specific chemicals into pore walls, (B) measuring the fluid flow through medium [4], and (C) statistical methods such as stereological, optical, and NMR analysis.

In the adsorption based methods, Methylene Blue Dye, as well as gas adsorption, has been used to determine the surface area of clay minerals for several decades [5]. Dogan et al. [6] measured the specific surface of clay minerals by the Brunauer, Emmett, and Teller (BET) method of adsorption of nitrogen gas. Fluid flow methods which are basically an application of Kozeny-Carman equation are widely treated in the literature. This equation is the most famous permeabilityporosity relation, which is widely used in the field of flow in porous media and is the starting point for many other permeability models $[7,8]$. The equation can be written as follows [4]:

$$
k=C_{0} \frac{\varphi^{3} /(1-\varphi)^{2}}{S^{2}},
$$

where $k$ is permeability, $C_{0}$ is a constant depending on pore shapes, $\varphi$ is porosity, and $S$ is the specific surface area of porous media.

Nuclear magnetic resonance (NMR) methods are also utilized widely to characterize rock's pore geometries. Pape et al. [9] developed a fractal analysis method for estimation of specific surface area of rocks using NMR data. The optical method which is based on statistical image processing has been developed by Chalkley [10]. The method is based on 
random tests on two- dimensional images from cross sections of synthetic porous medium [10]. In this regard a specified needle with length of $L$ is randomly thrown on the surface of cross section and the number of pores and grains on which the needle is laying should be counted [10]. The modified equation which estimates the specific surface of particles using Chalkley's method can be expressed as follows [1]:

$$
S=\frac{4 \varphi c}{L h}
$$

where $c$ is the number of particle perimeters intersected by the needle, $h$ is the number of pore spaces recumbent under the needle, $L$ is the needle length, and $\varphi$ is the porosity of porous medium.

Debye et al. [11] presented a method for estimation of specific surface of heterogeneous solids by measuring the X-ray scattering pattern from different materials. They tried to correlate the porosity of porous structure versus the specific surface of its particles and verified the results by measuring the specific surface using adsorption methods. Berryman [12] modified the method used by Debye et al. [11] by angular averaging between normal vectors of rock grain surface in order to estimate the specific surface value using scattering data of X-ray. Berryman and Blair [13] utilized scanning electron microscopy (SEM) to estimate porous medium porosity and specific surface by applying two-point correlation functions. They compared the results obtained from the KozenyCarman equation and experimental permeability measurement to adjust the two-point correlation function for finding the specific surface. Berryman and Blair [14] extended their work to consider tortuosity and electrical formation factor to calculate specific surface using data available for Berea sand stone. Furthermore, they discussed how to choose the optimum magnification of imaging in order to estimate the specific surface from rock cross sections.

Yu et al. [15] developed a fractal two-phase porous media model and estimated the specific surface areas in two and three dimensions. They expressed that the specific surface of porous media is a function of the fractal dimensions and microstructural parameters. In addition, they found that the $2 \mathrm{D}$ specific surface (specific perimeter) is in relation to the $3 \mathrm{D}$ specific surface, which is dependent on the grain size and fractal dimension.

$\mathrm{X}$-ray microtomographic imaging and visualization of rock material at the pore scale can give an important insight for understanding rock structure's properties. 3D images allow one to map in detail the pore and grain structure and interconnectivity of the porous medium [16]. Okabe and Blunt [17] utilized multiple-point statistics based on twodimensional thin sections as training images to generate geologically realistic $3 \mathrm{D}$ pore-space representations. As they stated, the thin section images can provide multiple-point statistics, which describe the statistical relation between multiple spatial locations and use the probability of occurrence of particular patterns. Using the generated 3D structure they predicted the absolute permeability of the sandstone sample using the effective medium theory and lattice Boltzmann simulation method.
Okabe and Blunt [18] used the approximated value of specific surface from $2 \mathrm{D}$ images to reconstruct $3 \mathrm{D}$ images of rock. In this regard, they tried to generate a $3 \mathrm{D}$ volume with the same specific surface as the estimated value from $2 \mathrm{D}$ images. A method of estimating the specific surface from 2D images has been previously developed by Adler et al. [19].

Politis et al. [20] presented a process-based and stochastic reconstruction method of porous media. Their method uses binary SEM images of rock to obtain a reconstruction method based on specific surface measurements. Blunt et al. [21] presented a vast field of experiences in the concept of pore scale imaging and modeling by providing a high resolution data bank of Micro-CT images of rock pieces. Mostaghimi et al. [22] utilized this data bank to present a suitable size of representative elementary volume (REV) for estimation of geometry-based properties such as porosity and specific surface area.

One main challenge in the application of multiple-point statistics to the simulation of three-dimensional (3D) blocks is the lack of suitable 3D training images. Comunian et al. [23] compared three methods to solve this challenge by attending (2D) training images: (a) the method based on aggregation of probabilities, (b) the method based on merging the lists obtained using the impala algorithm from diverse 2D training images and creating a list of compatible data events that is then used for the multiple-point statistics simulation, and (c) the method based on sequential simulations of $2 \mathrm{D}$ slices constrained by the conditioning data computed at the previous simulation steps.

In order to estimate the specific surface value of rock grains, cross-sectional images of different rocks have been utilized by coupling image analysis methods and statistical calculations. Initially, the specific surface of different rock structures has been calculated by computational methods with high precision using Micro-CT images provided by Blunt et al. [21]. Sahimi [24] stated that photomicrographs of polished sections of a sample porous medium with sufficient contrast between the pores and the matrix can be used to determine the specific surface of rock grains. Sahimi [24] stated that from the relation between $2 \mathrm{D}$ (surface) measurements and the properties of the 3D system, an estimate of specific surface can be obtained. The main objective in this study is to correlate the three-dimensional value of grain specific surface versus $2 \mathrm{D}$ characteristics of rock sections. The study here also presents a straightforward relationship between specific surface and the ratio of particle perimeter by its area in $2 \mathrm{D}$ images.

\section{Methodology}

One of the main challenges in extracting data from 2D images of porous medium is how to section the medium. This can be normally done by various angles and positions while the perpendicular orientation is more common. The true values of porosity and specific surface may not be visualized in sectional images because of limited field of view in two dimensions. In this work, the key parameter in estimation of specific surface is the ratio of grain particle perimeter to the area 


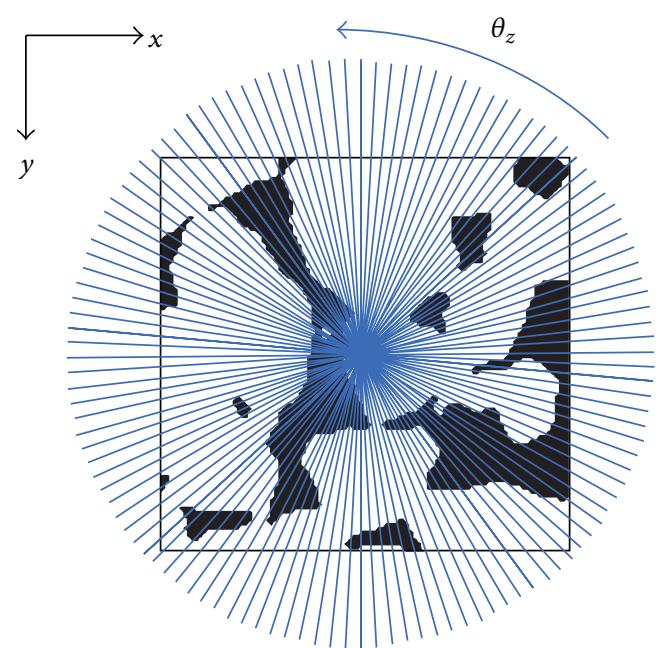

FIGURE 1: Schematic pattern of 180 degrees of cross sectioning in direction of $\theta_{z}$.

of each grain in $2 \mathrm{D}$ section called specific perimeter. The definition of specific perimeter can be expressed as

$$
\text { Specific Perimeter }=P_{s}=\frac{\text { Object Perimeter }}{\text { Object Surface Area }} \text {. }
$$

In order to examine the specific surface of grains in more detail, each 3D sample of rock has been gridded to smaller cubic parts. Calculation of specific surface from 3D images in each lattice requires counting the pixels of the image. This however depends on the image resolution. Estimation of specific perimeter requires a specified pattern in sectioning. Lock et al. [25] utilized an averaging approach for the range of 180 degrees of cross section angle in order to find mean pore size of rock images. A similar approach of cross sectioning is used in this work to consider various probabilities and complexities of grain structure. Figure 1 illustrates the pattern of cross sections in the plane of $x$ - and $y$-axis named as $\theta_{z}$ direction. The black and white parts lying under the cross lines present pores and grains of the rock, respectively. Each cross section indicates a $2 \mathrm{D}$ image which was analyzed by computer coding to find the specific perimeter. Next step is to average the data to upscale the results. Averaging specific perimeter in $\theta_{z}$ direction can be written as

$$
\left\langle P_{s}\right\rangle=\frac{\int_{0}^{\pi} P_{s}\left(\theta_{z}\right) d \theta_{z}}{\int_{0}^{\pi} d \theta_{z}}
$$

where $\left\langle P_{s}\right\rangle$ is normalized specific perimeter and $P_{s}\left(\theta_{z}\right)$ is a function that gives back the single value of specific perimeter for a cross section with an angle $\theta_{z}$. Here, $\left\langle P_{s}\right\rangle$ is the average of specific perimeter for all possible angles of cross sections. Figure 2 presents a 3D view of one grid from a binary image of Berea sandstone as one of the rock types investigated in the current work. Binary data are provided from Blunt et al.'s database [21].

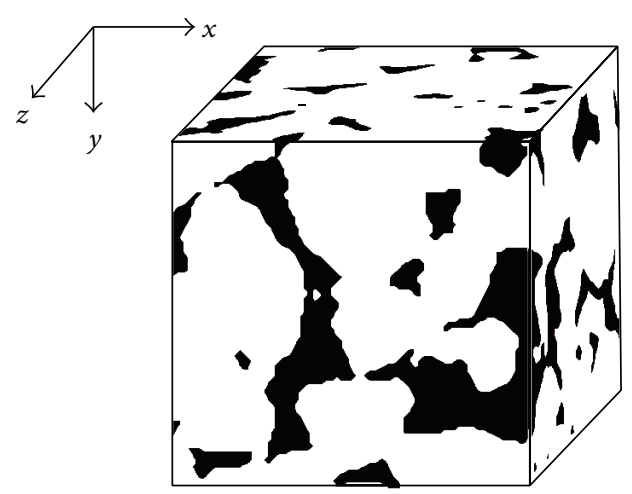

FIGURE 2: 3D view of sample binary image of Berea sandstone $(0.5 \mathrm{~mm})$.

Consequently, an averaged value for specific perimeter in each arbitrary section can be obtained as

$$
\left\langle P_{s}\right\rangle=\frac{\int_{0}^{\pi} P_{s}\left(\theta_{x}\right) d \theta_{x}+\int_{0}^{\pi} P_{s}\left(\theta_{y}\right) d \theta_{y}+\int_{0}^{\pi} P_{s}\left(\theta_{z}\right) d \theta_{z}}{\int_{0}^{\pi} d \theta_{x}+\int_{0}^{\pi} d \theta_{y}+\int_{0}^{\pi} d \theta_{z}} .
$$

The plane that contains each of $\theta_{x}, \theta_{y}$, and $\theta_{z}$ is clarified in Figure 3 . According to the integral definition, by simplifying the denominator, (6) changes to

$$
\left\langle P_{s}\right\rangle=\frac{\int_{0}^{\pi} P_{s}\left(\theta_{x}\right) d \theta_{x}+\int_{0}^{\pi} P_{s}\left(\theta_{y}\right) d \theta_{y}+\int_{0}^{\pi} P_{s}\left(\theta_{z}\right) d \theta_{z}}{3 \pi} .
$$

Although specific perimeter $\left(P_{s}\right)$ is a function of cross sectioning an angle, its dependency to sectioning angle cannot be formulized easily. Due to the unstructured and random shape of rock grains, the value of specific perimeter fluctuates randomly versus angle of cross section. Figure 4 shows the variations of specific perimeter for 6 different samples of Berea sandstone versus 90 degrees of cross section rotation. Apparently, no specific trend can be detected for describing the function's behavior. Also, there is a similar situation for one of the rock samples (Berea sandstone) in the different plane of angles labelled as $\theta_{x}, \theta_{y}$, and $\theta_{z}$. Figure 5 presents the changes of specific perimeter for different angles of cross section from 0 to 180 degrees for this sample. As clarified in Figure 1, in order to have an overall view of rock specific perimeter, all possible angles of cross section should be observed and recorded for each rock piece. As illustrated in Figure 5, the value of specific perimeter at $0^{\circ}$ is equal to its value at $180^{\circ}$, because of a complete rotation of the sectioning line around the sample.

Due to random behavior of specific perimeter for different angles (as visible in Figure 5), a numerical integration method (trapezoidal rule method) is used to solve (7). By estimation of the integrals using this method, the value of normalized specific perimeter has been obtained for each sample grid of rock. This calculation of integral is done using the developed computational code via MATLAB software for 


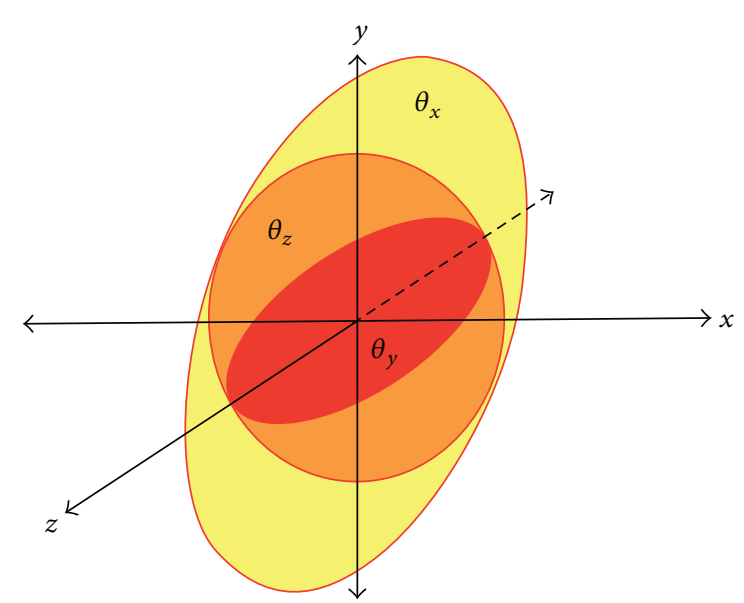

FIgURE 3: Clarification of angles and their plates for integral on cross sections.

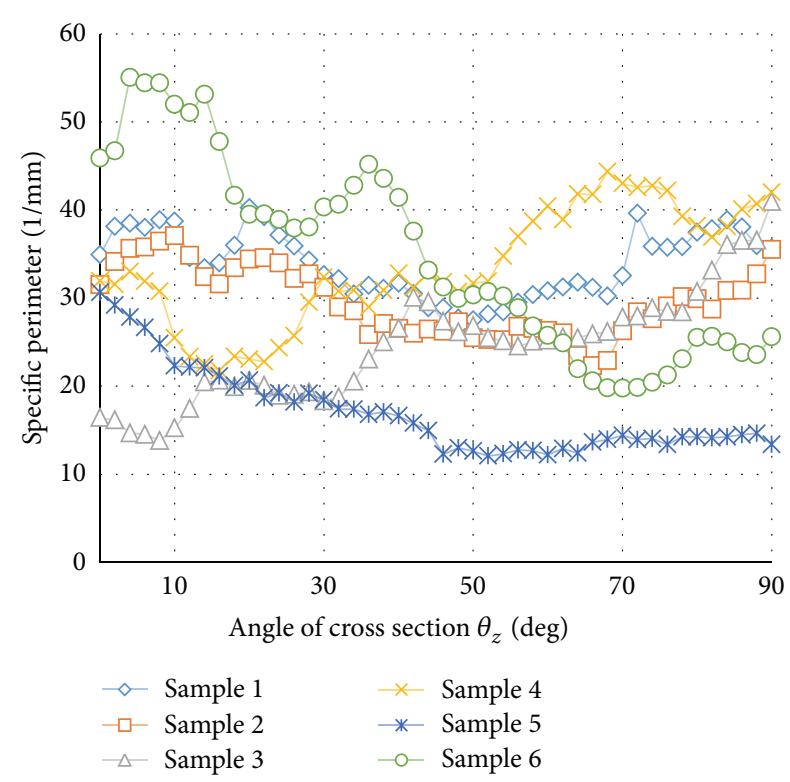

Figure 4: The variations of specific perimeter versus angle of cross section for 6 different samples of Berea sandstone.

each angle in each grid of different rock types that are provided in Figure 6.

In the next step, it has been found that there may be a meaningful relationship between specific perimeter and specific surface of a porous material. Considering the rock grains as perfect spheres, the ratio between the specific surface and normalized specific perimeter $\left\langle P_{s}\right\rangle$ can be stated as

$$
C_{g}=\frac{S}{\left\langle P_{s}\right\rangle}=\frac{4 \pi r^{2} /(4 / 3) \pi r^{3}}{2 \pi r / \pi r^{2}}=\frac{3}{2}
$$

Here, $r$ is the sphere radius and $C_{g}$ is Geometric Coefficient. This ratio can be used for representing the ideal porous medium with considering the fact that real grain structures do not fully resemble spherical structures.

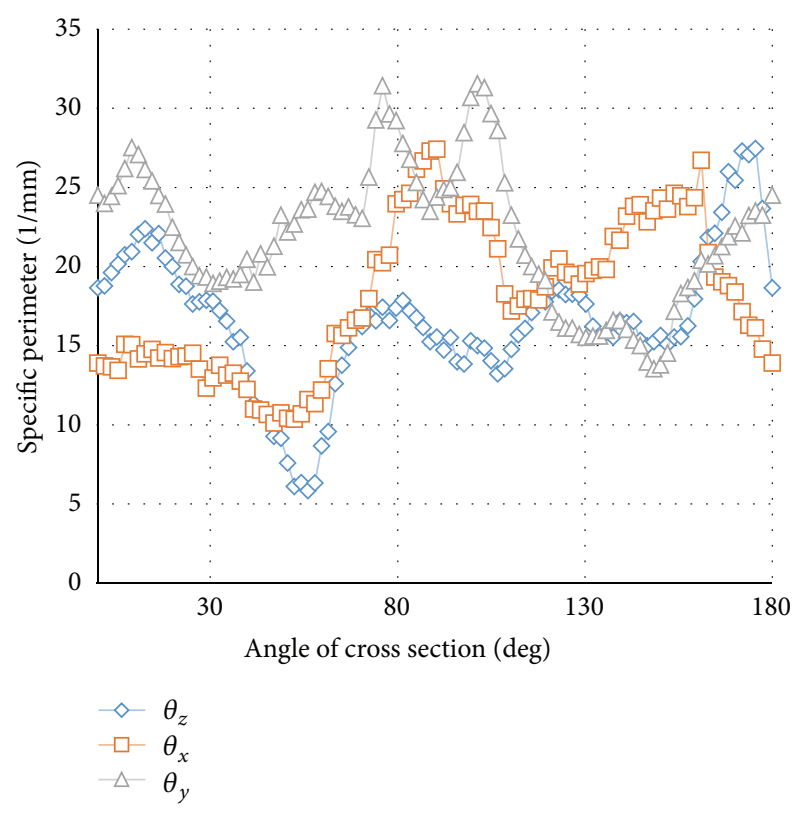

FIGURE 5: The fluctuation of specific perimeter for different planes of angles $\theta_{x}, \theta_{y}$, and $\theta_{z}$ in Berea sandstone.

\section{Results and Discussions}

Binary 2D images of different studied rocks are provided in Figure 6 . The black area represents the pore space in each image. The heterogeneity of carbonate samples is also visible in these images. The length at the bottom of each image shows the size of the square side.

In order to find the ratio between specific surface and normalized specific perimeter of porous materials, six different rock types are investigated. Berea sandstone as a well-known rock type shows almost uniform scattered data in estimation of Geometric Coefficient $\left(C_{g}\right)$. Figure 7 shows that the value of Geometric Coefficient varies versus normalized specific perimeter of $2 \mathrm{D}$ images for more than 500 samples. It can be concluded that most of the data points are almost centralized around an average value. Here, the value is about 1.37 for $C_{g}$.

The average $P_{s}$ and standard deviation for its range of changes have been calculated for six different rock types using the algorithm presented earlier in this paper. Table 1 presents the detailed results of a running model on several samples. The average and standard deviation for each recorded parameter are presented. The average of Geometric Coefficient for different samples does not vary significantly. This shows that there is a reliable ratio between the specific surface and specific perimeter of rock grains. Although the carbonate samples are significantly heterogeneous and the standard deviation of their parameters has relatively large values, $P_{s}$ does not change significantly.

As a key conclusion, the results of applying the present algorithm on 2184 rock samples from six different rock types leads one to declare an averaged value for Geometric Coefficient about 1.35 . Size of each cubic sample is fifty pixels which show the real size by multiplying image resolution. 


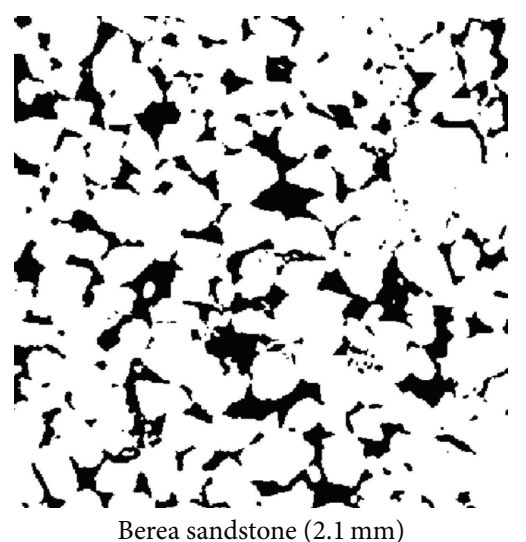

(a)

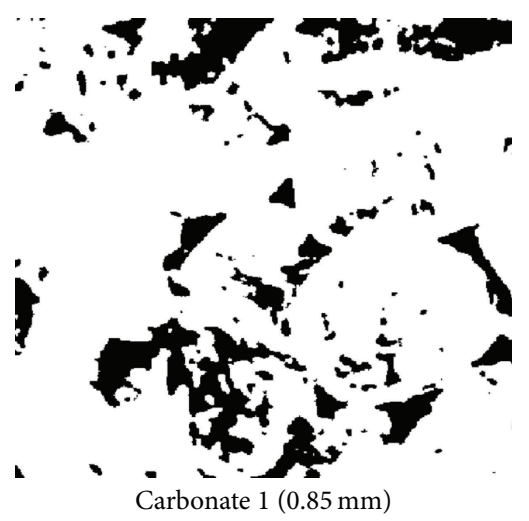

(d)

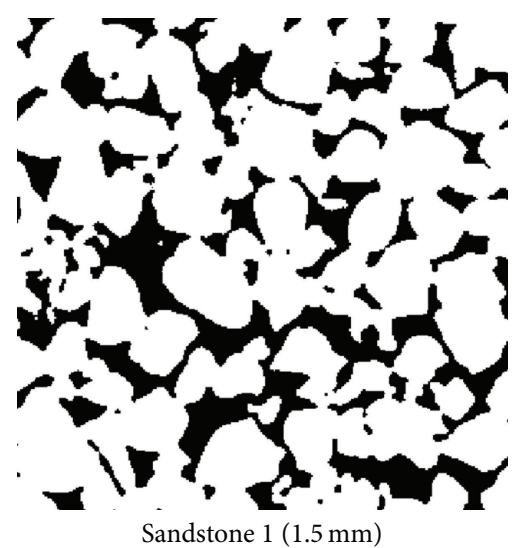

(b)

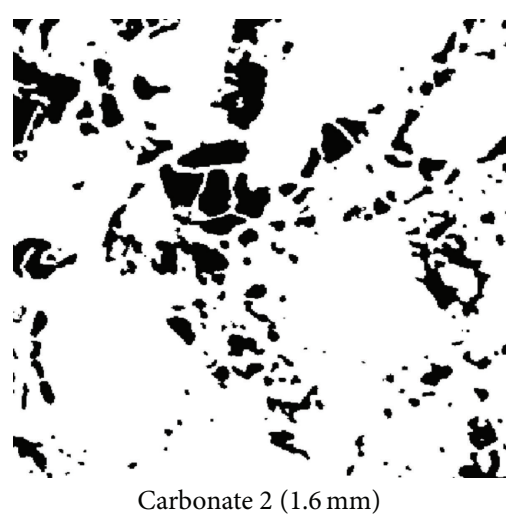

(e)

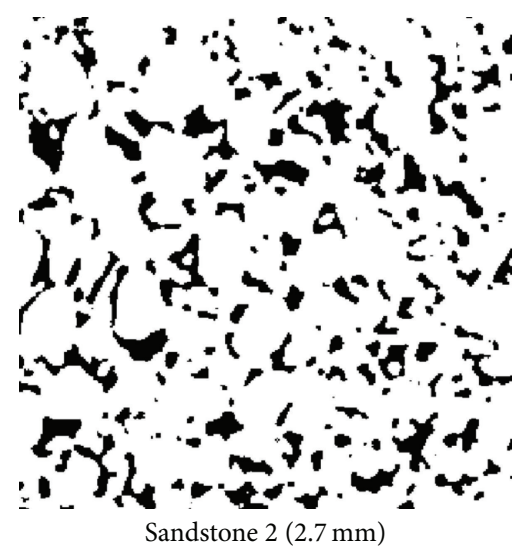

(c)

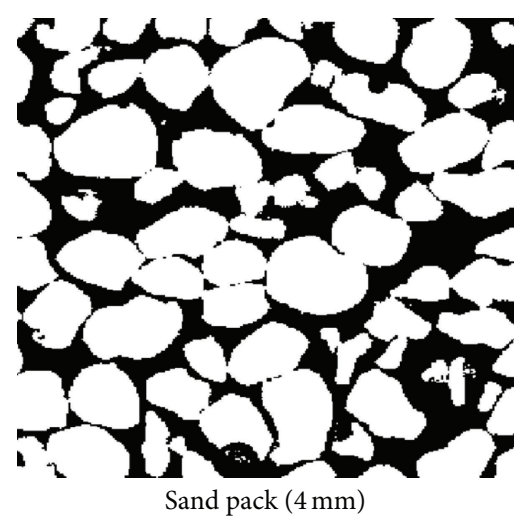

(f)

FIGURE 6: Images of used rock types and textures.

TABLE 1: Specific surface and perimeter and Geometric Coefficient $\left(C_{g}\right)$ calculated by model.

\begin{tabular}{|c|c|c|c|c|c|c|c|c|c|c|}
\hline \multirow{2}{*}{ Rock type } & \multirow{2}{*}{$\begin{array}{l}\text { Resolution } \\
(\mu \mathrm{m})\end{array}$} & \multirow{2}{*}{$\begin{array}{l}\text { Number of } \\
\text { Samples }\end{array}$} & \multicolumn{2}{|c|}{ Specific surface $\left(\mathrm{mm}^{-1}\right)$} & \multicolumn{2}{|c|}{ Specific perimeter $\left(\mathrm{mm}^{-1}\right)$} & \multicolumn{2}{|c|}{ Porosity } & \multicolumn{2}{|c|}{ Geometric Coefficient } \\
\hline & & & Average & $\begin{array}{l}\text { Standard } \\
\text { deviation }\end{array}$ & Average & $\begin{array}{l}\text { Standard } \\
\text { deviation }\end{array}$ & Average & $\begin{array}{l}\text { Standard } \\
\text { deviation }\end{array}$ & Average & $\begin{array}{l}\text { Standard } \\
\text { deviation }\end{array}$ \\
\hline Berea & 5.36 & 512 & 30.22 & 6.84 & 22.25 & 3.06 & 0.2 & 0.06 & 1.37 & 0.31 \\
\hline Sandstone 1 & 4.96 & 216 & 36.59 & 5.41 & 27.49 & 5.41 & 0.25 & 0.05 & 1.34 & 0.21 \\
\hline Sandstone 2 & 9.1 & 216 & 23.86 & 3.22 & 17.42 & 1.78 & 0.17 & 0.03 & 1.38 & 0.19 \\
\hline Carbonate 1 & 2.85 & 512 & 55.73 & 42.7 & 43.53 & 22.78 & 0.23 & 0.17 & 1.32 & 0.83 \\
\hline Carbonate 2 & 5.35 & 512 & 27.58 & 21.66 & 20.71 & 9.36 & 0.17 & 0.14 & 1.36 & 0.92 \\
\hline Sand pack & 10 & 216 & 22.09 & 3.39 & 16.51 & 1.77 & 0.33 & 0.05 & 1.35 & 0.20 \\
\hline
\end{tabular}

The same procedure used in the Figure 7 sample has been applied to the other five rock types and the values of Geometric Coefficient have been plotted. Figure 8 illustrates the scattering of Geometric Coefficient for two types of sandstones and one sand pack, the specifications of which are available in Table 1 . The ratio between specific surface and specific perimeter (Geometric Coefficient) for all three groups of samples is slightly changing around the value 1.4.

Figure 9 shows the severe fluctuation of Geometric Coefficient for two carbonate rocks. Apparently, it can be concluded that there is not any specific pattern in scattering data of those samples. Additionally, increasing the size of samples (representative elementary volume) does not indicate a symmetric pattern of scattering.

In order to evaluate the precision of the estimated specific surface, these values are plotted versus the measured specific surfaces for all six rock types. In this regard, hundred random cubes are chosen among each rock body and their specific surfaces have been estimated by multiplying the specific perimeter to 1.35. Figure 10 illustrates how data are scattered around the real values. The Coefficient of Determination which is also known as $R$-Squared indicates the precision of prediction. Here the value is about 0.63 which is reasonable considering the heterogeneity of samples and variety of rock 


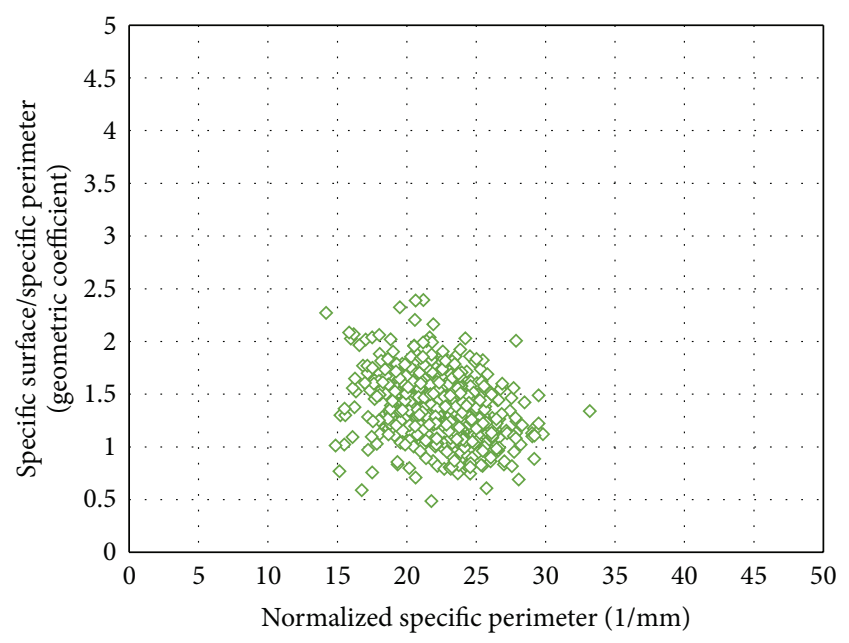

Figure 7: Plot of Geometric Coefficient versus normalized specific perimeter for Berea sandstone.

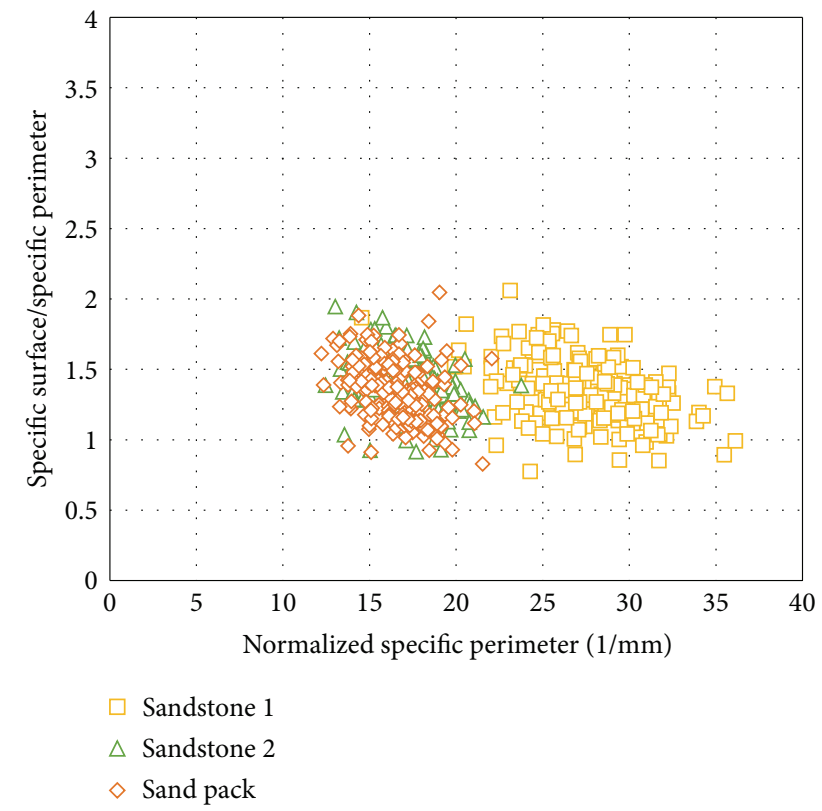

Figure 8: Geometric Coefficient for sandstone 1, sandstone 2, and sand pack samples versus normalized specific perimeter.

types. As it is visible, the carbonate samples show more diversity from the unit slope line. This indicates that the presented model cannot be used comprehensively to predict carbonates specific surface while it seems to be applicable for sandstones.

\section{Conclusions}

In this study, a Geometric Coefficient has been found to estimate the specific surface area $(S)$ of rock grains from $2 \mathrm{D}$ images. Normalized specific perimeter $\left\langle P_{s}\right\rangle$ is the averaged ratio between grains perimeter to their area in the images.

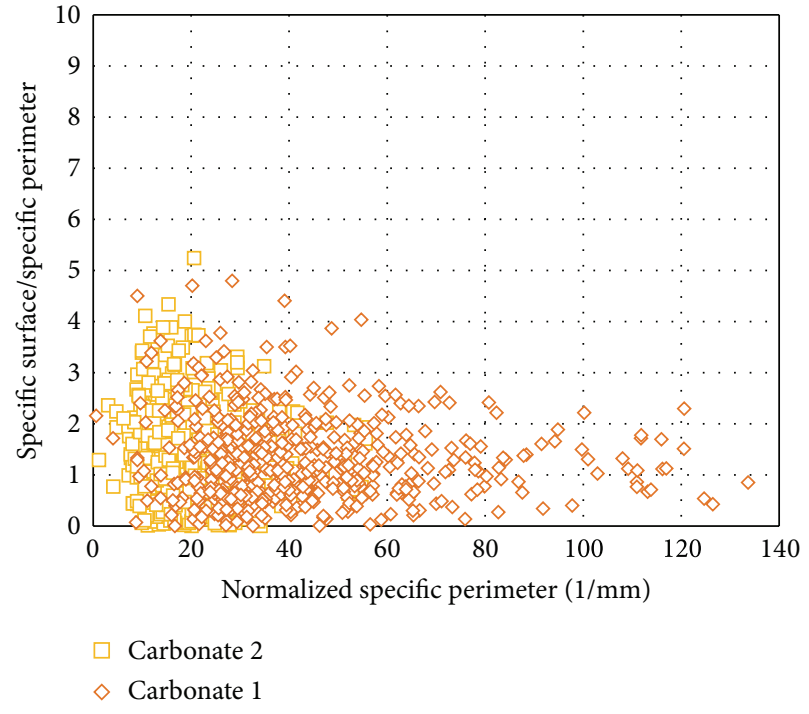

Figure 9: Geometric Coefficient for carbonate 1 and carbonate 2 samples versus normalized specific perimeter.

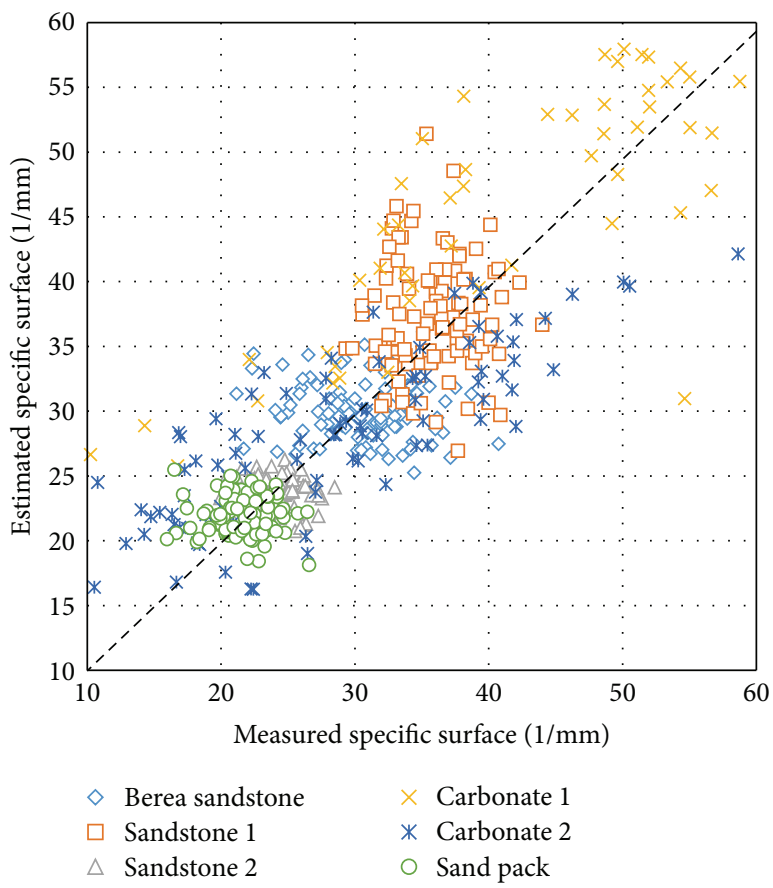

FIGURE 10: Predicted specific surface versus the measured values of 100 random samples for each of 6 rock types.

The Geometric Coefficient $\left(C_{g}\right)$ has been found to be about 1.35 for various types of rocks as follows:

$$
S=C_{g} \times\left\langle P_{s}\right\rangle=1.35 \times\left\langle P_{s}\right\rangle .
$$

Based on analysis in this study in order to estimate specific surface area of rock grains one can rely on calculating and averaging the specific perimeter from several 2D images and then multiply the normalized value by 1.35 . These $2 \mathrm{D}$ images may be obtained from thin section microscopy or 
any other photographical method which scans only the outer surface of the porous media. The results indicate that this approach is relatively robust for estimation of specific surface of sandstones. Using this approach is not recommended for carbonate rocks, and therefore future work is recommended.

Finally, the approach in this study can provide some preliminary observations of grain structures for modelling purposes when $3 \mathrm{D}$ data is not available.

\section{Nomenclature}

$C_{0}$ : Kozeny constant

$C_{g}:$ Geometric Coefficient

$P_{s}: \quad$ Specific perimeter

$\theta_{x}$ : Cross section angle in the plane perpendicular to axis $x$

$\theta_{y}: \quad$ Cross section angle in the plane perpendicular to axis $y$

$\theta_{z}$ : Cross section angle in the plane perpendicular to axis $z$

$\left\langle P_{s}\right\rangle$ : Normalized (averaged) specific perimeter

$h$ : Hits, number of pore spaces recumbent under the needle

$L: \quad$ Needle length

S: $\quad$ Specific surface

$c$ : $\quad$ Cuts, number of particle perimeters intersect by needle

$k$ : Permeability

$r: \quad$ Sphere radius

$\varphi$ : Porosity.

\section{Conflict of Interests}

The authors declare that there is no conflict of interests regarding the publication of this paper.

\section{References}

[1] R. E. Collins, Flow of Fluids through Porous Materials, PennWell Publishing, Tulsa, Okla, USA, 1961.

[2] F. A. Dullien, Porous Media: Fluid Transport and Pore Structure, Harcourt Brace Jovanovich Publishers, San Diego, Calif, USA, 1991.

[3] W. L. McCabe, Unit Operations of Chemical Engineering, Chemical Engineering Series, McGraw-Hill, 7th edition, 2005.

[4] A. Costa, "Permeability-porosity relationship: a reexamination of the Kozeny-Carman equation based on a fractal pore-space geometry assumption," Geophysical Research Letters, vol. 33, no. 2, 2006 .

[5] J. C. Santamarina, K. A. Klein, Y. H. Wang, and E. Prencke, "Specific surface: determination and relevance," Canadian Geotechnical Journal, vol. 39, no. 1, pp. 233-241, 2002.

[6] A. U. Dogan, M. Dogan, M. Omal, Y. Sarikaya, A. Aburub, and D. E. Wurster, "Baseline studies of The Clay Minerals Society source clays: specific surface area by Brunauer Emmett Teller (BET) method," Clays and Clay Minerals, vol. 54, no. 1, pp. 6266, 2006 .

[7] P. Xu and B. Yu, "Developing a new form of permeability and Kozeny-Carman constant for homogeneous porous media by means of fractal geometry," Advances in Water Resources, vol. 31, no. 1, pp. 74-81, 2008.

[8] N. Henderson, J. C. Brêttas, and W. F. Sacco, "A three-parameter Kozeny-Carman generalized equation for fractal porous media," Chemical Engineering Science, vol. 65, no. 15, pp. 44324442, 2010.

[9] H. Pape, J. E. Tillich, and M. Holz, "Pore geometry of sandstone derived from pulsed field gradient NMR," Journal of Applied Geophysics, vol. 58, no. 3, pp. 232-252, 2006.

[10] H. W. Chalkley, "Method for the quantitative morphologic analysis of tissues," Journal of the National Cancer Institute, vol. 4, no. 1, pp. 47-53, 1943.

[11] P. Debye, H. R. Anderson Jr., and H. Brumberger, "Scattering by an inhomogeneous solid. II. the correlation function and its application," Journal of Applied Physics, vol. 28, no. 6, pp. 679683, 1957.

[12] J. G. Berryman, "Relationship between specific surface area and spatial correlation functions for anisotropic porous media," Journal of Mathematical Physics, vol. 28, no. 1, pp. 244-245, 1987.

[13] J. G. Berryman and S. C. Blair, "Use of digital image analysis to estimate fluid permeability of porous materials: application of two-point correlation functions," Journal of Applied Physics, vol. 60, no. 6, pp. 1930-1938, 1986.

[14] J. G. Berryman and S. C. Blair, "Kozeny-Carman relations and image processing methods for estimating Darcy's constant," Journal of Applied Physics, vol. 62, no. 6, pp. 2221-2228, 1987.

[15] B. Yu, J. Cai, and M. Zou, "On the physical properties of apparent two-phase fractal porous media," Vadose Zone Journal, vol. 8, no. 1, pp. 177-186, 2009.

[16] M. A. Knackstedt, S. Latham, M. Madadi, A. Sheppard, T. Varslot, and C. Arns, "Digital rock physics: 3D imaging of core material and correlations to acoustic and flow properties," The Leading Edge, vol. 28, no. 1, pp. 28-33, 2009.

[17] H. Okabe and M. J. Blunt, "Prediction of permeability for porous media reconstructed using multiple-point statistics," Physical Review E, vol. 70, no. 6, Article ID 066135, p. 066135/10, 2004.

[18] H. Okabe and M. J. Blunt, "Pore space reconstruction using multiple-point statistics," Journal of Petroleum Science and Engineering, vol. 46, no. 1-2, pp. 121-137, 2005.

[19] P. M. Adler, C. G. Jacquin, and J. A. Quiblier, "Flow in simulated porous media," International Journal of Multiphase Flow, vol. 16, no. 4, pp. 691-712, 1990.

[20] M. G. Politis, E. S. Kikkinides, M. E. Kainourgiakis, and A. K. Stubos, "A hybrid process-based and stochastic reconstruction method of porous media," Microporous and Mesoporous Materials, vol. 110, no. 1, pp. 92-99, 2008.

[21] M. J. Blunt, B. Bijeljic, H. Dong et al., "Pore-scale imaging and modelling," Advances in Water Resources, vol. 51, pp. 197-216, 2013.

[22] P. Mostaghimi, M. J. Blunt, and B. Bijeljic, "Computations of absolute permeability on micro-CT images," Mathematical Geosciences, vol. 45, no. 1, pp. 103-125, 2013.

[23] A. Comunian, P. Renard, and J. Straubhaar, "3D multiple-point statistics simulation using 2D training images," Computers \& Geosciences, vol. 40, pp. 49-65, 2012.

[24] M. Sahimi, Flow and Transport in Porous Media and Fractured Rock, Wiley-VCH, 2012.

[25] P. A. Lock, X. Jing, R. W. Zimmerman, and E. M. Schlueter, "Predicting the permeability of sandstone from image analysis of pore structure," Journal of Applied Physics, vol. 92, no. 10, pp. 6311-6319, 2002. 

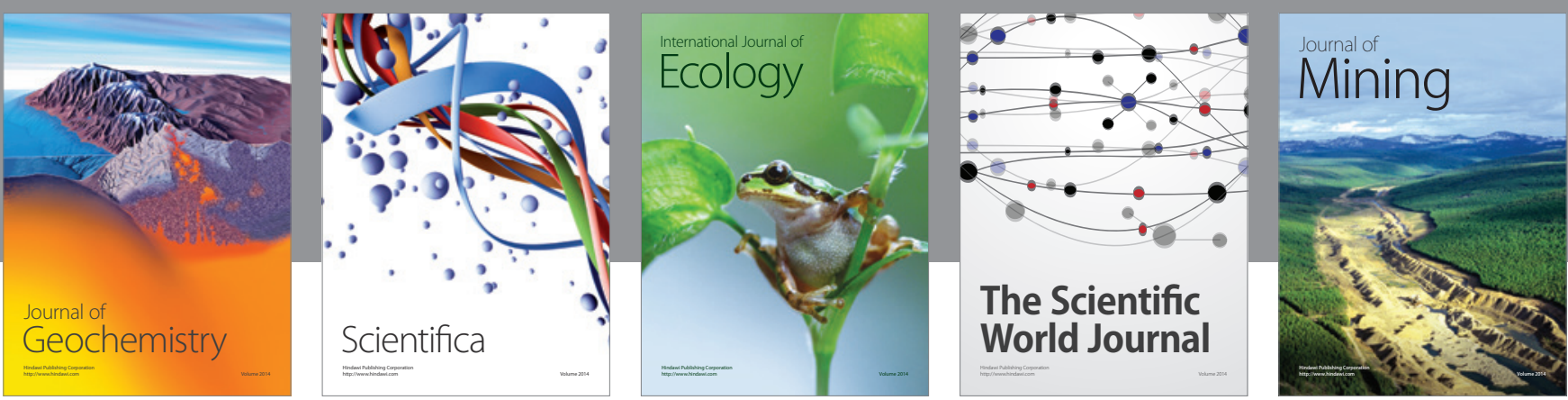

The Scientific World Journal
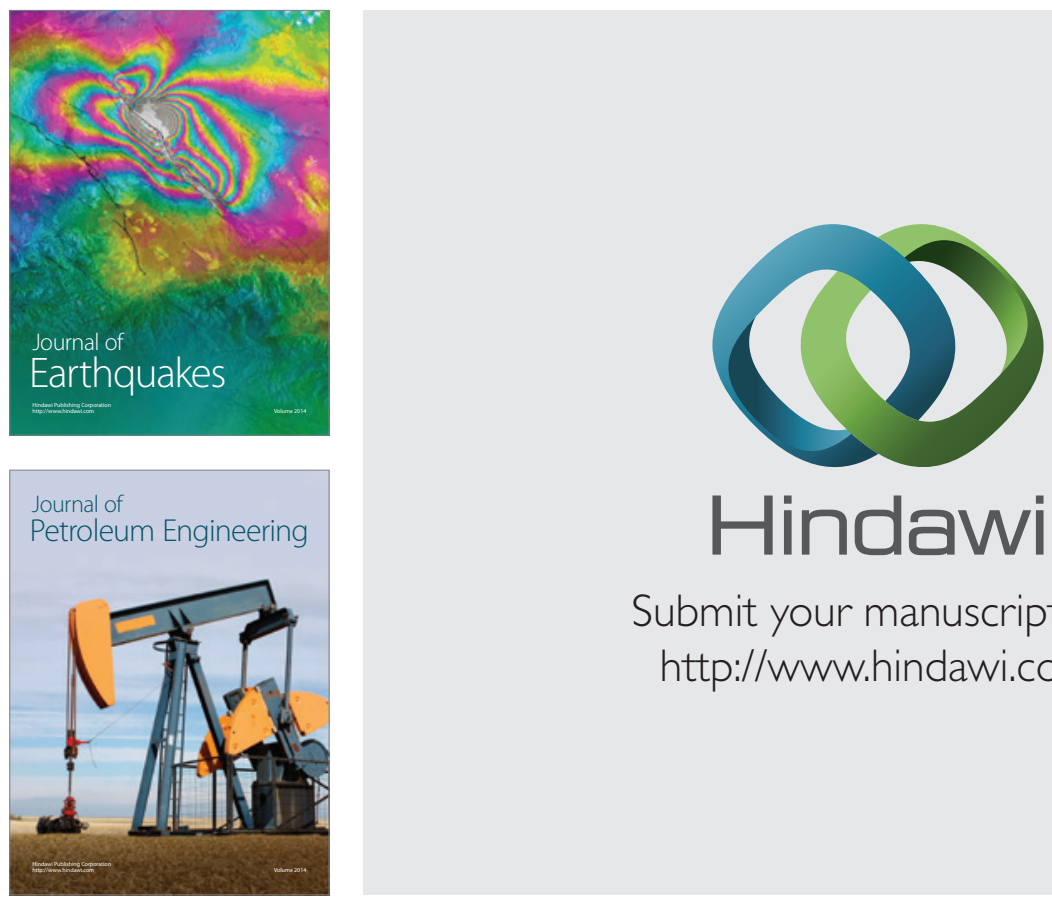

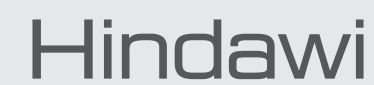

Submit your manuscripts at

http://www.hindawi.com
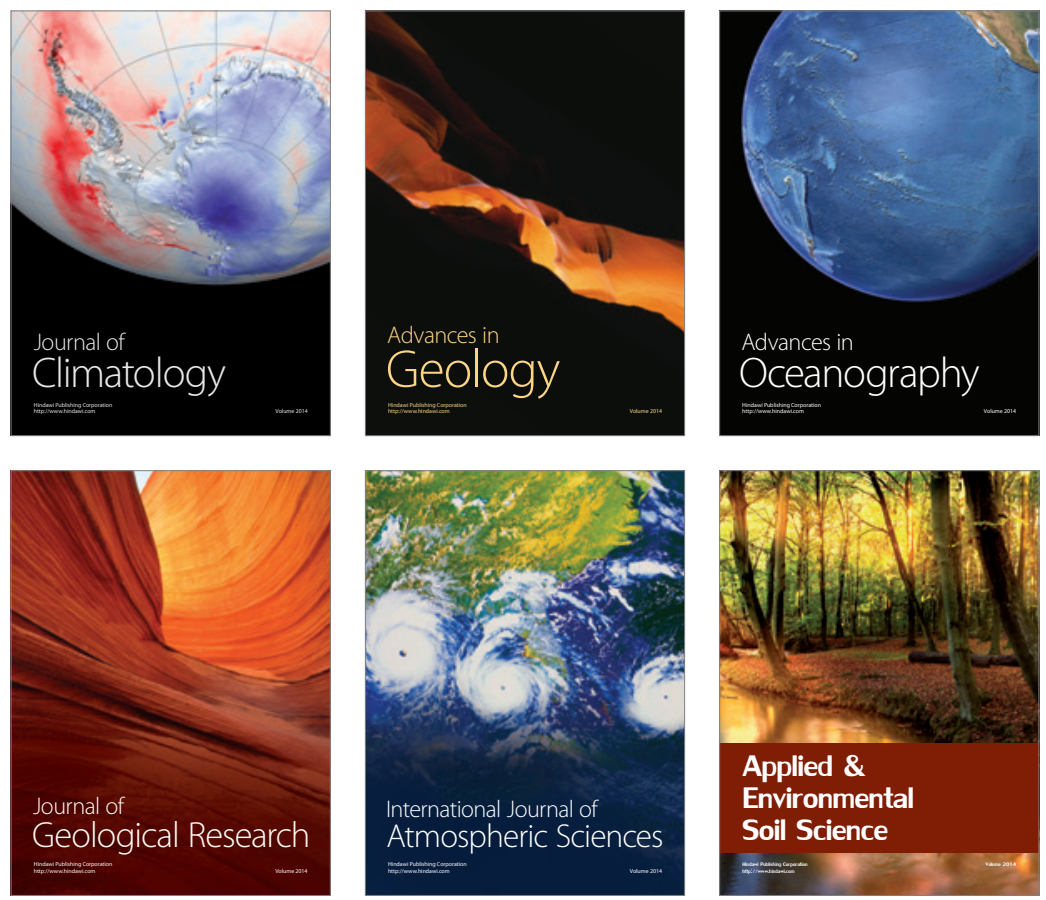
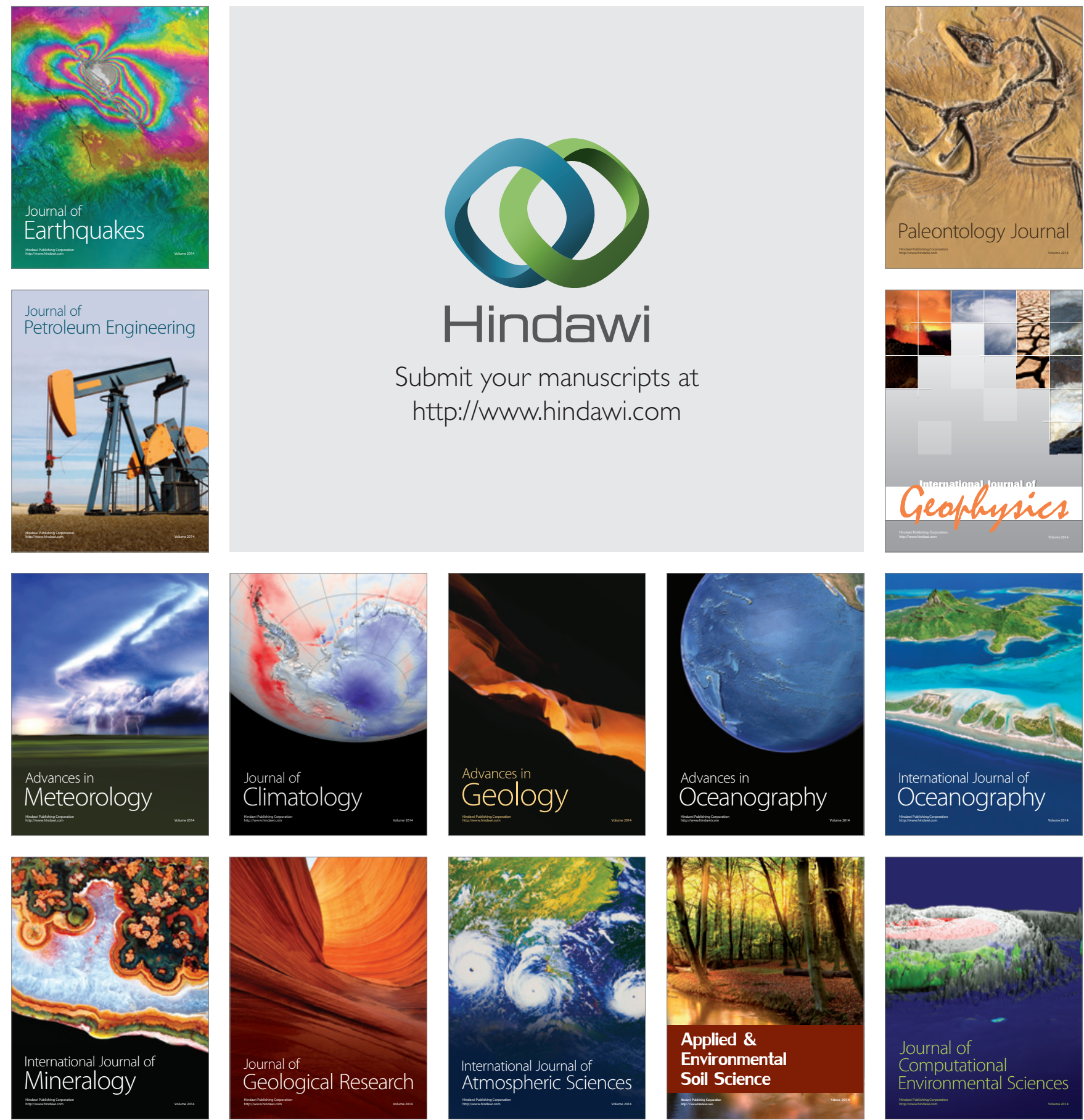